\title{
A Diagnostic Algorithm for the Detection of Clostridium difficile-Associated Diarrhea
}

\author{
Özlem Yoldaş ${ }^{1}$, Mustafa Altındiş ${ }^{2}$, Davut Cufalı ${ }^{3}$, Gülşah Aşık ${ }^{3}$, Recep Keşli ${ }^{3}$ \\ ${ }^{1}$ Clinical Microbiology Laboratory, Türkan Özilhan Bornova State Hospital, İzmir, Turkey \\ ${ }^{2}$ Department of Medical Microbiology, Sakarya University Faculty of Medicine, Sakarya, Turkey \\ ${ }^{3}$ Department of Medical Microbiology, Afyon Kocatepe University Faculty of Medicine, Afyon, Turkey
}

Background: Clostridium difficile is a common cause of hospital-acquired diarrhea, which is usually associated with previous antibiotic use. The clinical manifestations of $C$. difficile infection (CDI) may range from mild diarrhea to fulminant colitis. Clostridium difficile should be considered in diarrhea cases with a history of antibiotic use within the last 8 weeks (community-associated $\mathrm{CDI}$ ) or with a hospital stay of at least 3 days, regardless of the duration of antibiotic use (hospital-acquired CDI). Aims: This study investigated the frequency of CDI in diarrheic patients and evaluated the efficacy of the triple diagnostic algorithm that is proposed here for C. difficile detection.

Study Design: Cross-sectional study.

Methods: In this study, we compared three methods currently employed for $C$. difficile detection using 95 patient stool samples: an enzyme immunoassay (EIA) for toxin A/B (C. diff Toxin A+B; Diagnostic Automation Inc.; Calabasas, CA, USA), an EIA for glutamate dehydrogenase (GDH) (C. DIFF CHEK-60TM, TechLab Inc.; Blacksburg, VA, USA), and a polymerase chain reaction (PCR)-based assay (GeneXpert ${ }^{\mathbb{B}}$ C. difficile; Cepheid, Sunnyvale, CA, USA) that detects C. difficile toxin genes and conventional methods as well. In this study, $50.5 \%$ of the patients were male, 50 patients were outpatients, 32 were from inpatient clinics and 13 patients were from the intensive care unit.

Results: Of the 95 stool samples tested for GDH, 28 were positive. Six samples were positive by PCR, while nine samples were positive for toxin A/B. The hypervirulent strain NAP-1 and binary toxin was not detected. The rate of occurrence of toxigenic $C$. difficile was $5.1 \%$ in the samples. Cefaclor, ampicillin-sulbactam, ertapenem, and piperacillin-tazobactam were the most commonly used antibiotics by patients preceding the onset of diarrhea. Among the patients who were hospitalized in an intensive care unit for more than 7 days, $83.3 \%$ were positive for CDI by PCR screening. If the PCR test is accepted as the reference: $C$. difficile Toxin A/B ELISA sensitivity and specificity were $67 \%$ and $94 \%$, respectively, and GDH sensitivity and specificity were $100 \%$ and $75 \%$, respectively.

Conclusion: Tests targeting $C$. difficile toxins are frequently applied for the purpose of diagnosing CDI in a clinical setting. However, changes in the temperature and reductant composition of the feces may affect toxin stability, potentially yielding false-negative test results. Therefore, employment of a GDH EIA, which has high sensitivity, as a screening test for the detection of toxigenic strains, may prevent false-negative results, and its adoption as part of a multistep diagnostic algorithm may increase accuracy in the diagnosis of CDIs.

Keywords: Clostridium difficile, antibiotic-associated diarrhea, toxin A/B, glutamate dehydrogenase, PCR assay $\left(\right.$ GeneXpert $\left.^{\circledR}\right)$

This study was presented at the $114^{\text {th }}$ General Meeting of American Society for Microbiology, 17-20 May 2014, Boston, USA.

Address for Correspondence: Dr. Mustafa Altındiş, Department of Medical Microbiology, Sakarya University Faculty of Medicine, Sakarya, Turkey

Phone: +905326615263 e-mail: maltindis@sakarya.edu.tr

Received: 23.02.2015 Accepted: $28.08 .2015 \cdot$ DOI: 10.5152/balkanmedj.2015.15159

Available at www.balkanmedicaljournal.org 
Clostridium difficile colonization can vary in severity from being asymptomatic to causing self-limiting diarrhea or pseudomembranous colitis. Clostridium difficile isolates are able to survive on abiotic surfaces in a healthcare setting through endospore formation, leading to difficulties in eradication and increased transmission. Upon increased colonization of the gut, toxigenic strains of $C$. difficile can cause infections. Hospital-acquired $C$. difficile epidemics can spread among patients, who become infected by healthcare professionals or contaminated instruments. In addition to healthcare-associated cases, community-acquired infections have recently increased in number. Toxigenic strains of $C$. difficile produce exotoxin $\mathrm{A}(\mathrm{Tcd} \mathrm{A})$ and exotoxin $\mathrm{B}(\mathrm{TcdB})$. A binary toxin (CDT) is frequently observed in hypervirulent $C$. difficile strains associated with the increased severity of $C$. difficile infection (CDI) such as the NAP-1 strain. CDT belongs to a family of binary ADP-ribosylating toxins consisting of two separate toxin components: CDTa, an ADPribosyltransferase that modifies actin, and $\mathrm{CDTb}$, which binds to host cells and translocates CDTa into the cytosol. The prevalence of the binary toxin in CDI is $1.6-20.8 \%$ (1). While the $C$. difficile carriage rate in healthy adults is $1-3 \%$, hospitalization is an important risk factor that increases the risk of colonization. After hospitalization, the frequency of asymptomatic colonization increases to $20-30 \%$, especially in elderly patients (2-4).

Clostridium difficile diagnostic testing employs different clinical microbiology methods with varying degrees of sensitivity, including molecular methods, chromatographic techniques, and assays for the presence of TcdA/B using culturecytotoxicity or immunological methods. A final diagnosis of CDI is based on the presence of free toxin in patient stool samples and the detection of associated cytopathic effects in cell culture. However, many of these methods are technically demanding and time-consuming; thus, many laboratories prefer enzyme-based immunological methods that detect glutamate dehydrogenase (GDH) or antigens and toxins. However, the latter approach has much lower sensitivity. Polymerase chain reaction (PCR)-based methods that identify the agent directly from clinical samples have recently been introduced and are currently in use in many laboratories (5).

The most widely used laboratory test is still the identification of toxins A and B via an enzyme-linked immunosorbent assay (ELISA) (6). However, difficulties arise in the standardization of screening methods due to the range and dissimilarity of the diagnostic tests used in laboratories. This study aimed to identify the presence of $C$. difficile in samples from patients with diarrhea using multiple techniques in an effort to suggest a screening algorithm with different approaches and diagnostic tests.

\section{MATERIALS AND METHODS}

\section{Study, subjects, and participation}

In this cross-sectional study, stool samples from 95 patients admitted to a university hospital were used. Each patient had been prescribed antibiotics within 8 weeks preceding the study and was admitted with a complaint of diarrhea. The study population was comprised of 45 inpatients and 50 outpatients. Ethics committee approval was received for this study from the ethics committee of Afyon Kocatepe University Faculty of Medicine and written informed consent was obtained from patients who participated in this study.

\section{Methods}

Based on macroscopic examinations, 95 soft, watery, bloody and/or mucous-containing stool samples were tested for $C$. difficile. Shaped and solid stool samples were not included in the study. For the purpose of direct microscopic examination, specimens were prepared using physiological saline and placed on slides with cover slips. The samples were then evaluated for the presence of erythrocytes, leukocytes, parasitic cysts, and eggs as well as yeast cells and/or pseudohyphae formation. To test for common enteropathogenic bacteria, all samples were plated onto eosin methylene blue agar and Salmonella Shigella (SS) agar and inoculated into selenite F medium. After $4 \mathrm{~h}$, the samples were subcultured on SS agar and incubated at $37^{\circ} \mathrm{C}$ in an aerobic environment for $24-48 \mathrm{~h}$.

\section{Isolation and detection of $\boldsymbol{C}$. difficile}

The culture of $C$. difficile from the stool samples was performed using cycloserine-cefoxitin fructose agar selective solid media. The plate was incubated in Hi gas-pak jar at $37^{\circ} \mathrm{C}$ for $48 \mathrm{~h}$ using BD GasPak EZ Anaerobe container system with indicator or in individual sachets (BD GasPak ${ }^{\mathrm{TM}} \mathrm{EZ}$ Gas Generating Pouch Systems; New Jersey, USA). On CCFA, circular, yellow, fimbriate colonies that were $4 \mathrm{~mm}$ in size or larger, of Gram-positive bacilli with subterminal oval spores and a horse stable odor were presumptively identified as $C$. difficile.

Colonies morphologically resembling the organism were tested by latex agglutination with the Oxoid $C$. difficile Test Kit (DR 1107A), UK according to the manufacturer's instructions. Briefly, saline suspension of the suspected colony was mixed with Oxoid $C$. difficile Latex Reagent on the reaction card. Appearance of agglutination was examined for a maximum of two minutes, employing appropriate negative and positive controls.

\section{GDH, Toxin Testing by EIA and PCR}

The presence of GDH, a membrane-associated enzyme of C. difficile, in the fecal samples was determined using an ELI- 
SA (C. DIFF CHEK-60 ${ }^{\mathrm{TM}}$, TechLab Inc.; Blacksburg, VA). To identify the presence of TcdA and B in the fecal samples, an ELISA kit for toxin $\mathrm{A} / \mathrm{B}(C$. diff Toxin $\mathrm{A}+\mathrm{B}$; Diagnostic Automation Inc., Calabasas, $\mathrm{CA}$ ) and a $C$. difficile PCR kit (GeneXpert ${ }^{\circledR}$ C. difficile; Cepheid, Sunnyvale, CA) were used. Following amplification by real-time PCR, DNA extraction and PCR were performed using a single-use cartridge. This system detected sequences specific to toxin B $(t c d B)$, binary toxin $(c d t)$, and $t c d C \Delta 117$. It also allowed for the determination of toxin-producing $C$. difficile and the NAP-1 strain. The PCR test was adopted as a reference assay for tests investigating toxins. Positive and negative control samples were included for each of the parameters tested.

\section{Statistical analysis}

The data were analyzed using SPSS 17.0 for Windows (SPSS Inc.; Chicago, IL, USA). A Chi-square test was applied to assess the statistical significance of the data.

\section{RESULTS}

Of the samples tested, $50.5 \%$ were obtained from male patients while $49.5 \%$ were from female patients. In total, $12 \%$ of the patients were $2-17$ years old, $21 \%$ were $18-39$ years old, $27.3 \%$ were $40-64$ years old, and $44.2 \%$ were $>65$ years old.

In total, $52.6 \%, 33.7 \%$, and $13.7 \%$ of the samples were collected from inpatients, outpatients, and intensive care unit patients, respectively. The presence of common enteropathogenic agents such as Salmonella sp., Shigella sp., Campylobacter sp., and E. coli $\mathrm{O} 157$ could not be determined in these patients using conventional bacterial identification methods. Any patient who tested positive for these pathogens was excluded from the study.

Positive PCR results for GDH were obtained in all of the toxigenic $C$. difficile-positive patients, but the Tcd ELISA yielded negative results in two patients. Similarly, two patients who tested positive for both GDH and toxins in their respective ELISA assays, showed negative results according to the PCR-based method. There were also three patients who tested negative for GDH and had negative PCR results but who showed the presence of toxins according to the ELISA. The proportions of positive results from the GDH test and the toxin screens are shown in Table 1.

In this study, toxigenic $C$. difficile was identified by PCR in $6.8 \%$ of the patients. Whereas the toxin ELISA had sensitivity, specificity, positive predictive, and negative predictive values of $67 \%, 94 \%, 21.4 \%$ and $95.5 \%$, respectively, GDH had $100 \%, 75 \%, 21.4 \%$, and $100 \%$ values, respectively, compared to the PCR-based method.

Of those patients who tested positive for toxigenic $C$. diffcile by PCR, two had previously been treated with piperacillintazobactam, one with ertapenem and ampicillin+sulbactam, one had a history of ertapenem and colistin use, one had a history of cefaclor use, and one had been treated with ampicillin+sulbactam (each for 10-14 days). The statistical significance of the effects of antibiotic use on future CDI was investigated using a chi-square test $(\mathrm{p}=0.582)$. Demographic data for the toxigenic $C$. difficile-positive patients identified by PCR are given in Table 2 .

In our study, hospitalized patients had a higher rate of $C$. difficile colonization in all of the tests we applied. The distribution of positive results by patient history at the time of hospitalization is shown in Table 3. PCR screening of the patient samples did not reveal any NAP-1- or binary toxin-positive strains in this study.

TABLE 1. A comparison of positive rate of GDH and the other tests for C. difficile diarrhea

\begin{tabular}{lccccccc}
\hline & \multicolumn{2}{c}{ Toxin ELISA } & \multicolumn{2}{c}{ PCR } & \multicolumn{3}{c}{ Culture for $C$. difficile } \\
& Positive & Negative & Positive & Negative & Positive & Negative & Total \\
\hline GDH Positive & 6 & 22 & 6 & 22 & 10 & 18 & 28 \\
GDH Negative & 3 & 64 & 0 & 67 & 0 & 67 & 67 \\
Total & 9 & 86 & 6 & 89 & 10 & 85 & 95 \\
\hline
\end{tabular}

GDH: glutamate dehydrogenase; PCR: polymerase chain reaction

TABLE 2. The epidemiological data of toxigenic $C$. difficile PCR-positive patients

\begin{tabular}{|c|c|c|c|c|c|c|c|}
\hline Sex & Age & Comorbidities & $\begin{array}{c}\text { Length of stay } \\
\text { in hospital }\end{array}$ & Antibiotics used & $\begin{array}{l}\text { Duration of } \\
\text { antibiotic use }\end{array}$ & $\begin{array}{l}\text { C. difficile } \\
\text { toxin ELISA }\end{array}$ & GDH \\
\hline Women & 30 & $\begin{array}{c}\text { Thoracic intramedullary } \\
\text { mass + pregnancy }\end{array}$ & 30 days & Ampicillin/Sulbactam & 8 days & + & + \\
\hline Women & 29 & bloodstream infection & 21 & Sefaclor & 10 days & - & + \\
\hline Man & 82 & $\begin{array}{l}\text { Epilepsy + Pneumonia } \\
+ \text { Acute Renal Failure }\end{array}$ & 37 days & $\begin{array}{l}\text { Ampicillin/Sulbactam } \\
\text { Ertapenem }\end{array}$ & $\begin{array}{l}14 \text { days } \\
10 \text { days }\end{array}$ & + & + \\
\hline Man & 83 & $\begin{array}{l}\text { Acute Renal Failure }+ \\
\text { Cerebro vascular stroke }\end{array}$ & 58 days & $\begin{array}{l}\text { Ertapenem } \\
\text { Colistine }\end{array}$ & $\begin{array}{l}14 \text { days } \\
3 \text { days }\end{array}$ & + & + \\
\hline Women & 51 & Gastrointestinal bleeding & 10 days & Piperacillin/tazobactam & 10 days & + & + \\
\hline Man & 73 & Brucellosis & 7 days & Piperacillin/tazobactam & 7 days & + & + \\
\hline
\end{tabular}

GDH: glutamate dehydrogenase; PCR: polymerase chain reaction 
TABLE 3. The distribution of positive rate between inpatient and community associated $C$. difficile diarrhea

\begin{tabular}{lccc}
\hline & GDH \% & PCR \% & Toxin ELISA \% \\
\hline $\begin{array}{l}\text { Inpatient (n: 50) } \\
\text { Community associated }\end{array}$ & 46.7 & 11.1 & 17.8 \\
$\begin{array}{l}\text { C. difficile diarrhea (n: 45) } \\
\text { Total (n: 95) }\end{array}$ & 14 & 2 & 2 \\
\hline
\end{tabular}

GDH: glutamate dehydrogenase; PCR: polymerase chain reaction

\section{DISCUSSION}

The current gold standard approach for the diagnosis of CDIs is cell culture, cytotoxicity tests, and neutralization tests (7). These methods require experienced personnel and equipment, are often time-consuming, difficult to standardize, and have a high incidence of false-positives due to the presence of proteases in stool. As such, new screening methods are required (8). The algorithm best suited for a rapid and accurate diagnosis is controversial, and many laboratories use tests that look for toxins. Tests such as ELISAs and the card test, which are used to search for toxins A and B, have low sensitivity, although they are rapid and inexpensive. However, these toxins can also be inactivated by changes in temperature and fecal proteases, resulting in a potential increase in false-negatives $(9,10)$.

In 2005, our previously study (11) investigated the presence of toxigenic $C$. difficile in stool samples collected from 91 patients, of which 45 were outpatients and 46 were inpatients, as well as from hospital staff ( 7 intensive care and 20 food service personnel). In that study, culture and latex agglutination tests with specific antisera were performed and the presence of either toxin A or B was investigated using an ELISA or the latex agglutination assay. Based on their ELISA results, $15.5 \%$ of the outpatients and $17.1 \%$ of the inpatients were positive for CDI, while no hospital personnel tested positive. When the toxin ELISA results were compared to those of the culture-latex agglutination test, the toxin A latex method had a sensitivity of $30.7 \%$ compared to $100 \%$ for the toxin ELISA. In that study, the number of cases with $C$. difficile toxin present was comparable to that in other studies of hospital- and community-acquired cases. Hospitalization is an important risk factor for CDI. In present study, toxin ELISA positivity was higher in the hospitalized patient group.

Another important risk factor for CDI is advanced age ( $>65$ years) (9). In our study, $66.7 \%$ of the toxigenic $C$. difficilepositive patients were older than 55 years of age. Consistently in the literature, the most frequently used antibiotics in patients with CDI are beta-lactams. In our study, this was not the case: the most frequently used antibiotics in diarrhea cases were from different antibiotic families $(\mathrm{p}=0.582)$.

Since the sensitivity of the ELISA and card test methods used to investigate the presence of toxins A and B is low, a
GDH ELISA is often involved in diagnostic algorithms. In addition to the increased sensitivity of the assay, GDH antigens appear both in toxigenic and non-toxigenic strains, allowing for the detection of both variants in patient samples. Upon the identification of antigen in stool samples using a GDH ELISA, the presence of $C$. difficile should be confirmed using an alternate method because it has low specificity despite its high sensitivity (12).

The Society for Healthcare Epidemiology of America and the Infectious Diseases Society of America recommend testing amorphous stool samples from suspected $C$. difficile cases only for patients suffering from diarrhea, excluding cases of ileus. Testing fecal samples from asymptomatic patients is not recommended. It is believed that although fecal culture is important and sensitive for the purpose of epidemiological studies, it is not suitable for use in clinical practice because it is time-consuming and the identification of toxigenic strains requires specialized experience and equipment. Toxin ELISAs are rapid but have low sensitivity; therefore, they are suboptimal diagnostic tools. Latex agglutination kits identifying GDH have a sensitivity of 58-68\% and specificity of 94-98\%, while ELISA-based kits have $85-95 \%$ sensitivity and 89-99\% specificity. The use of GDH in two-step diagnostic algorithms together with confirmatory tests is an adoptable strategy. It is recommended to interpret the result as $C$. difficile-negative if GDH assays are negative, and to proceed with a confirmation test if GDH is detected. Since PCR-based tests are fast, sensitive, and specific, they can be adopted as a new diagnostic approach, although additional data are required for these tests before they are put into routine clinical use (13).

In a study of 114 stool samples performed by LaSala et al. (14), $24(12 \%)$ were positive for CDI using a GDH test, a PCR-based test, and a toxin-based ELISA, 22 (19\%) were positive using GDH- and PCR-based tests, 7 (33\%) were positive using a GDH-based test, and 1 was positive according to a GDH test and toxin-based ELISA. The authors concluded that using only a stand-alone toxin test may give false-negative results. Fenner et al. (15) made use of a two-step C. difficile diagnostic algorithm for 1468 stool samples and used PCR to resolve any inconsistencies. They reported 187 (12.7\%) GDHpositive samples and $69(36.9 \%)$ toxin A/B positive-samples. Of the GDH-negative patients, ten had toxin positivity, and a slight bacterial burden was identified in five patients using PCR. In total, $52.9 \%$ of the GDH- and toxin-positive patients had a positive toxigenic culture, although the culture sensitivity was reported to be low.

Crobach et al. (6) investigated 18 different diagnostic methods from 1991-2008; 13 different ELISAs in 34 toxin A/B studies, 4 GDH methods in 14 ELISA GDH studies, and 1 PCR method in 4 PCR studies. In $85 \%$ of the studies, the gold 
standard cytotoxicity test served as the reference test. Using a two-step algorithm, a 5\% prevalence of CDI was calculated for 10000 patients. Among the patients, 9541 were truly negative, 432 were truly positive, 68 had a false-negative result, and 49 had a false-positive result. Application of the high-sensitivity GDH method as the initial screening test with a high-specificity toxin test as confirmation, and screening for the presence of $C$. difficile in diarrhea cases with no presence of other enteropathogenic agents is recommended. Williamson et al. (16) analyzed stool samples from 7106 patients, allocating 3100 of the samples to the GDH assay group and 4006 samples to a two-step procedure using PCR together with a GDH assay. According to their results, $4.7 \%$ of the samples showed the presence of $C$. difficile based on testing with only the GDH assay. This number increased to $9.9 \%$ when the two-step algorithm was used. According to our results, utilization of a GDH test with high sensitivity as a rapid screening test appears to be an important step in preventing false-negatives. Babady et al. (17) compared PCR and GDH culture cytotoxicity using 560 stool samples and found that the sensitivity of GDH culture cytotoxicity and PCR combined (100\%) was higher than that of GDH-positive culture cytotoxicity assays alone (57\%).

The detection of toxins via latex agglutination tests may produce false-positive results due to cross-reactions between toxigenic and/or nontoxigenic strains of $C$. difficile and Peptostreptococcus anaerobius and Bacteroides asaccharolyticus (18). The lower sensitivity and specificity of the toxin-based ELISA and its inconsistencies with the GDH method observed in this study point to PCR as a beneficial test for the confirmation of GDH-positive patients. GDH-positive, toxin-negative, PCR-positive results should be correlated with the clinical symptoms of the patient. Other causes of diarrhea should be ruled out before seeking a $C$. difficile diagnosis. However, positive PCR results cannot distinguish between asymptomatic colonization and a true CDI. A potential triple diagnostic algorithm is illustrated in Figure 1. Table 4 provides a summary of the $C$. difficile test results.

An additional study investigated current $C$. difficile diagnostic algorithms and determined the number of positive tests in 170 hospitals in the United Kingdom between 2009 and 2010. The study determined that the majority of hospital laboratories (70\%) use toxin $\mathrm{A} / \mathrm{B}$ tests $(6 \%$ use the card test and $64 \%$ use ELISAs), while $3.6 \%$ of hospitals use cell cytotoxicity neutralization tests. Only one laboratory used PCR as a screening method. Moreover, $19 \%$ of the laboratories were reported to have adopted a two-step algorithm, only performing the second step if the results of the initial screen were positive. An additional 5\% of laboratories used a triple algorithm comprised of a GDH test, toxin ELISA, and PCR, while 5\% were using GDH and toxin A/B combination kits (19).

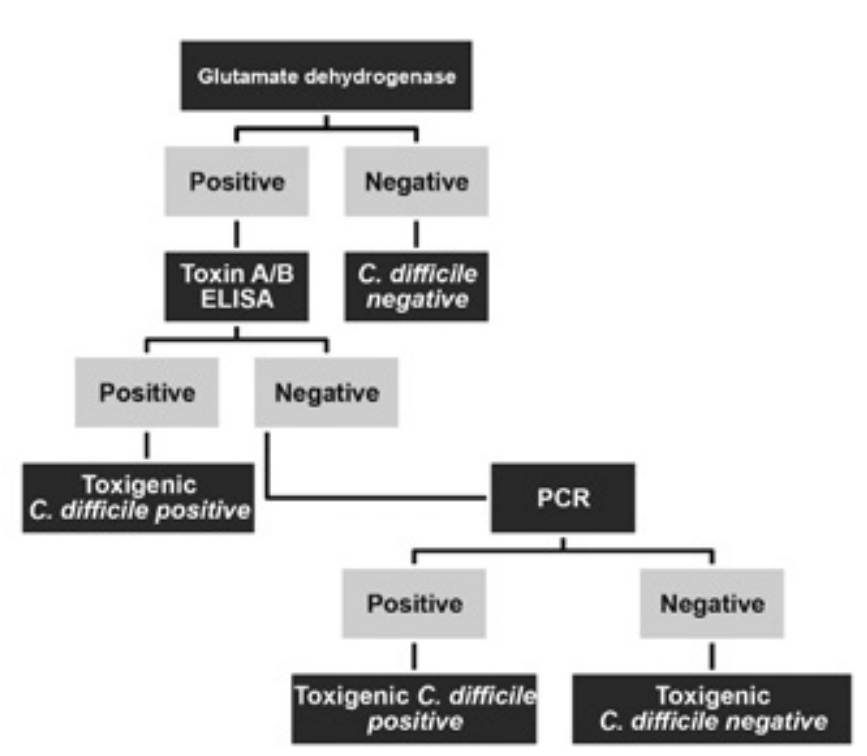

FIG. 1. Diagnostic algorithm of Clostridium difficile

TABLE 4. Interpretation of $C$. difficile tests results

\begin{tabular}{lcc}
\hline GDH/NAAT & Toxin ELISA & Comments \\
\hline Positive & Positive & C. difficile Positive \\
Positive & Negative & Potential carriers of $C$. difficile \\
Negative & Negative & There is no $C$. difficile \\
& & (Probably other pathogens) \\
\hline
\end{tabular}

GDH: glutamate dehydrogenase; NAAT: nucleic acid amplification testing

C. difficile infection is increasing in prevalence and severity. Major risk factors include previous antibiotic use, advanced age, stays in a hospital or healthcare setting, and (possibly) the use of proton pump inhibitors. Most cases are related to antibiotic use, but sporadic cases can occur in otherwise healthy individuals with no known risk factors (20). Korac et al. (21) demonstrated that fluoroquinolones and third-generation cephalosporins were used most often among CDI patients who had been treated previously with antibiotics. Until recently, fluoroquinolones were widely used for surgical prophylaxis in Serbia, which may explain why more than $50 \%$ of the patients in our study had received them before CDI occurred. In this study, several CDI patients identified by PCR had previously been treated with antibiotics: two with piperacillin-tazobactam, one with ertapenem and ampicillin+sulbactam, one with ertapenem and colistin, one with cefaclor, and one with ampicillin+sulbactam, each for a duration of 10-14 days (Table 2).

The risk factors for $C$. difficile-associated diarrhea are wellknown and clinicians are recommended to evaluate patients according to these risk factors. Problems in making an accurate diagnosis may occur despite the availability of rapid and 
reliable tests due to differences in the specificity of the various screens available (22).

In making a CDI diagnosis, stool samples may have blood present if severe colitis has occurred, but grossly bloody stools are unusual. Additionally, fecal leukocytes are present in about half of cases. Currently used stool assays for $C$. difficile, from the most to least sensitive are: stool culture, GDH enzyme immunoassays (EIAs), RT-PCR, toxin A/B EIAs, and latex agglutination assays $(23,24)$.

The culture of $C$. difficile from stool is the most sensitive test, but it is labor-intensive; results may be delayed by $48-96$ h. This method also often yields false-positives due to the presence of non-toxigenic strains. However, stool culture is important for epidemiological studies such as ribotyping and examinations of antibiotic susceptibility (25).

The GDH EIA is both a sensitive and specific test for CDI (85-100\% and $87-98 \%$, respectively). Latex agglutination assays are an alternative method for detecting $C$. difficile GDH; however, these methods are significantly less sensitive (4859\%) and less specific (95-96\%) than EIAs.

In our study cohort, sensitivity, specificity, positive predictive value, and negative predictive value were $67 \%, 94 \%$, $21.4 \%$ and $95.5 \%$ for toxin EIA and $100 \%, 75 \%, 21.4 \%$, and $100 \%$ for GDH, respectively. The PPV of $21.4 \%$ for GDH alone confirmed the need to pursue additional testing of GDHpositive/toxin or PCR negative specimens to resolve these specimens as positive or negative for $C$. Difficile toxin. Zheng et al. (26) reported that the Techlab C. diff Chek-60 GDH assay had good sensitivity compared to CYT testing of $92 \%$, but it had a low specificity of $89.1 \%$ and poor positive predictive value (PPV) of 57.7\%.

RT-PCR, which is used to detect $C$. difficile toxin genes, could be considered an alternative gold standard test owing to its specificity, but it is a comparatively expensive assay to perform. Currently, cell culture toxin neutralization tests are the gold standard for identifying CDIs, but they are less sensitive than PCR or toxigenic culture in patients presenting with diarrhea. Costs, sensitivity and specificity of molecular diagnostic tests vary. The costs of material and labor for $C$. difficile Toxin A B, cytotoxin Assay, GDH, GDH/toxin 2 step algorithm and nucleic acid amplification testing (NAAT) were 6, 25, 5-10, 8-14, and 25-48 USD, respectively (25). Similarly, toxin EIAs are among the most commonly used screening methods in laboratories, in part due to their high specificity. However, the sensitivity of these assays is considerably lower (70-80\%) than that of many of the other methods described here.

Many laboratories still use stand-alone toxin tests to diagnose CDI, and there is no standardization between healthcare facilities in terms of accepted diagnostic algorithms. In this study, we investigated three diagnostic tests to determine their respective sensitivities and specificities and proposed a new diagnostic algorithm for use in clinical settings. When the GDH EIA method is administered as a screening test with a toxin ELISA or card test as the confirmatory secondary screen, it increases the sensitivity and allows for the evaluation of possible false-positive results (27). The investigation of toxigenic $C$. difficile using PCR offers high sensitivity and specificity, but it is perhaps too expensive to be introduced widely into clinics. The simultaneous identification of 027/NAP-1 strains of $C$. difficile is an important advantage for epidemiological studies, particularly in countries where this hypervirulent strain is common. Clostridium difficile ribotype 176 may be misinterpreted as ribotype 027 by GeneXpert ${ }^{\circledR}$ C. difficile (Cepheid). Therefore, further molecular analysis such as ribotyping based on capillary electrophoresis is needed to better differentiate between $C$. difficile ribotypes 027 and 176 such that appropriate steps can be taken at the local and national levels to prevent outbreaks (28). In many places, C. difficile surveillance is not yet sufficient; thus, the number of outbreaks is increasing. Adopting a triple diagnostic algorithm and performing GDH tests and ELISAs with the confirmation of inconsistent results by PCR would enable clinicians to make a more accurate diagnosis.

Ethics Committee Approval: Ethics committee approval was received for this study from the ethics committee of Afyon Kocatepe University Faculty of Medicine.

Informed Consent: Written informed consent was obtained from patients who participated in this study.

Peer-review: Externally peer-reviewed.

Author contributions: Concept - M.A.; Design - M.A., Ö.Y.; Supervision - M.A.; Resource - M.A., Ö.Y.; Materials - Ö.Y., D.Ç.; Data Collection \&/or Processing - M.A., Ö.Y.; Analysis \&/or Interpretation - M.A., Ö.Y.; Literature Search - M.A., Ö.Y.; Writing - M.A., Ö.Y.; Critical Reviews - M.A., G.A., R.K.

Conflict of Interest: No conflict of interest was declared by the authors.

Financial Disclosure: The authors declared that this study has financial support by Afyon Kocatepe Üniversitesi Bilimsel Araştırma Projeleri Koordinasyon Birimi (AKU BAPK).

\section{REFERENCES}

1. Thielman NM, Wilson KH: Antibiotic-associated colitis. In Principles and Practice of Infectious Diseases. $7^{\text {th }}$ press. Benett (ed) Mandell GL, JE Dolin R. (eds), Churchill Livingstone, New York 2010. [CrossRef] 
2. Bagdasarian N, Rao K, Malani PN. Diagnosis and treatment of Clostridium difficile in adults: a systematic review. JAMA 2015;313:398-408. [CrossRef]

3. Rodriguez C, Korsak N, Taminiau B, Avesani V, Van Broeck J, Delmée $\mathrm{M}$, et al. Clostridium difficile infection in elderly nursing home residents. Anaerobe 2014;30:184-7. [CrossRef]

4. Tunçcan OG, Ulutan F, Karakuş R. The frequency of Clostridium difficile toxin in neutropenic and non-neutropenic patients with antibiotic-associated diarrhea and analysis of the risk factors. Mikrobiyol Bul 2008;42:573-83.

5. Freeman J, Bauer MP, Baines SD, Corver J, Fawley WN, Goorhuis B, et al. The Changing Epidemiology of Clostridium difficile Infections. Clin Microbiol Rev 2010;529-49. [CrossRef]

6. Crobach MJ, Dekkers OM, Wilcox MH, Kuijper EJ. European Society of Clinical Microbiology and Infectious Diseases (ESCMID): data review and recommendations for diagnosing Clostridium difficile-infection (CDI). Clin Microbiol Infect 2009;15:1053-66. [CrossRef]

7. Merz CS, Kramer C, Forman M, Gluck L, Mills K, Senft K, et al. Comparison of four commercially available rapid enzyme immunassays with cytotoxin assay for detection of Clostridium difficile toxin(s) from stool specimens. J Clin Microbiol 1994;32:1142-7.

8. Alcalá L, Sánchez-Cambronero L, Catalán MP, Sánchez-Somolinos M, Peláez MT, Marín M, et al. Comparison of three commercial methods for rapid detection of Clostridium difficile toxin A and B from fecal specimens. J Clin Microbiol 2008;46:3833-5. [CrossRef]

9. Sloan LM, Duresko BJ, Gustafson DR, Rosenblatt JE. Comparison of real time PCR for detection of the tcdC gene with four toxin immunassays and culture in diagnosis of Clostridium difficile infection. J Clin Microbiol 2008;46:1996-2001. [CrossRef]

10. Altindis M, Usluer S, Ciftçi H, Tunç N, Cetinkaya Z, Aktepe OC. Investigation of the presence of Clostridium difficile in antibiotic associated diarrhea patients by culture and toxin detection methods. Mikrobiyol Bul 2007;41:29-37.

11. Oldfield EC. Clostridium difficile-associated diarrhea: risk factors, diagnostic methods, and treatment. Rev Gastroenterol Disord 2004;4:186-95.

12. Zheng L, Keller SF, Lyerly DM, Carman RJ, Genheimer CW, Gleaves CA, et al. Multicenter evaluation of a new screening test that detects Clostridium difficile in fecal specimens. J Clin Microbiol 2004;42:3837-40. [CrossRef]

13. Cohen SH, Gerding DN, Johnson S, Kelly CP, Loo VG, McDonald LC, et al; Society for Healthcare Epidemiology of America; Infectious Diseases Society of America. Clinical Practice Guidelines for Clostridium difficile Infection in Adults: 2010 Update by the Society for Healthcare Epidemiology of America (SHEA) and the Infectious Diseases Society of America (IDSA). Infect Control Hosp Epidemiol 2010;31:5. [CrossRef]

14. LaSala PR, Svensson AM, Mohammad AA, Perrotta PL. Comparison of Analytical and Clinical Performance of Three Methods for Detection of Clostridium difficile. Arch Pathol Lab Med 2012;136:527-31. [CrossRef]
15. Fenner L, Widmer AF, Goy G, Rudin S, Frei R. Rapid and reliable diagnostic algorithm for detection of Clostridium difficile. J Clin Microbiol 2008;46:328-30. [CrossRef]

16. Williamson DA, Basu I, Freeman J, Swager T, Roberts SA. Improved detection of toxigenic $\mathrm{C}$. difficile using the Cepheid Xpert C. difficile assay and impact on C. difficile infection rates in tertiary hospital: A double edged sward. Am J Infect Control 2013;41:270-2. [CrossRef]

17. Babady NE, Stiles J, Ruggiero P, Khosa P, Huang D, Shuptar S, et al. Evaluation of Cepheid Xpert C. difficile epi assay for diagnosis of C. difficile infection and typing of the NAP1 strain at a cancer hospital. J Clin Microbiol 2010;48:4519-24. [CrossRef]

18. Lyerly DM, Ball DW, Toth J, Wilkins TD. Characterization of cross-reactive proteins detected by Culturette Brand Rapid Latex Test for Clostridium difficile. J Clin Microbiol 1988;26:397400 .

19. Goldenberg SD, French GL. Diagnostic testing for C. difficile: a comprehensive survey of laboratories in England. J Hosp Infect 2011;79:4-7. [CrossRef]

20. Surawicz CM. Clostridium difficile infection: risk factors, diagnosis and management. Curr Treat Options Gastroenterol 2015;13:121-9. [CrossRef]

21. Korac M, Milosevic I, Markovic M, Popovic N, Ilic M, Markovic A, et al. Clostridium difficile infection: a Serbian single-center experience. J Infect Dev Ctries 2015;19;9:136-40. [CrossRef]

22. Kilic A. Clostridium difficile Infection: Epidemiology, Risk Factors, Pathogenesis, Clinical Features, Diagnosis and Therapy. Mikrobiyol Bul 2013;47:556-66. [CrossRef]

23. Khanna S, Pardi DS, Aronson SL, Kammer PP, Orenstein R, St Sauver JL, et al. The epidemiology of community-acquired Clostridium difficile infection: a population-based study. Am J Gastroenterol 2012;107:89-95. [CrossRef]

24. Sloan LM, Duresko BJ, Gustafson DR, Rosenblatt JE. Comparison of real-time PCR for detection of the tcdC gene with four toxin immunoassays and culture in diagnosis of Clostridium difficile infection. J Clin Microbiol 2008;46:1996-2001. [CrossRef]

25. Juneau C, Mendias EN, Wagal N, Loeffelholz M, Savidge T, Croisant $\mathrm{S}$, et al. Community-Acquired Clostridium Difficile Infection: Awareness and Clinical Implications. J Nurse Pract 2013;9:1-6. [CrossRef]

26. Zheng, L, Keller SF, Lyerly DM, Carman RJ, Genheimer CW, et al. Multicenter evaluation of a new screening test that detects Clostridium difficile in fecal specimens. J Clin Microbiol 2004;42:3837-40. [CrossRef]

27. Debast SB, Bauer MP, Kuijper EJ; European Society of Clinical Microbiology and Infectious Diseases. European Society of Clinical Microbiology and Infectious Diseases: update of the treatment guidance document for Clostridium difficile infection. Clin Microbiol Infect 2014;20:1-26. [CrossRef]

28. Krutova M, Matejkova J, Nyc OC. Difficile ribotype 027 or 176? Folia Microbiol (Praha) 2014;59:523-6. [CrossRef] 